\title{
A Beam Scanning-Based Indoor Localization System using Light Emitting Diodes
}

\author{
Muhammad Saadi $^{1, \mathrm{a}}$, Yan Zhao ${ }^{2, \mathrm{~b}, *}$, Oumair Naseer ${ }^{1, \mathrm{c}}$, and Lunchakorn Wuttisttikulkij ${ }^{3, \mathrm{~d}}$ \\ 1 Department of Electrical Engineering, University of Central Punjab, Lahore, Pakistan \\ 2 International School of Engineering, Faculty of Engineering, Chulalongkorn University, Bangkok, \\ Thailand \\ 3 Department of Electrical Engineering, Faculty of Engineering, Chulalongkorn University, Bangkok, \\ Thailand \\ E-mail: amuhammadsaadi@gmail.com, byan.z@chula.ac.th (Corresponding author), \\ coumair.naseer@ucp.edu.pk, dlunchakorn.w@chula.ac.th
}

\begin{abstract}
In this paper, an indoor localization system using light emitting diodes (LEDs) is presented. Location of an object is determined by scanning two LEDs at pre-defined angles which are modulated at different frequencies. During scanning process, for each LED, two maximum values of received signal strength (RSS) are logged and this information is used to compute the position of an object. Time division multiplexing (TDM) is used to incorporate angle information at the receiver. A bicubic spline interpolation is applied to estimate the position of an object. Simulations are executed for a typical indoor environment with dimensions of $\left(3 \times 3 \times 2 \mathrm{~m}^{3}\right)$ and results are validated using experiments. The experimental results confirm that proposed beam scanning approach can be used to determine the location of an object precisely.
\end{abstract}

Keywords: Indoor localization, light emitting diodes, beam scanning, received signal strength.

ENGINEERING JOURNAL Volume 20 Issue 3

Received 18 March 2016

Accepted 4 May 2016

Published 19 August 2016

Online at http://www.engj.org/

DOI:10.4186/ej.2016.20.3.197 


\section{Introduction}

Localization, also known as positioning, is a process of determining the spatial position of an object or a person [1]. Location based services (LBSs), is a popular area of research nowadays because of its wide spread applications in daily life. Outdoor localization systems mostly rely on the global positioning system (GPS), however, this system is not suited for indoor localization mainly because of poor satellite coverage, signal attenuation, electromagnetic (EM) interference, multipath effect and obstacles [2]. For an indoor environment, visible light positioning system (VLPS) based on light emitting diodes (LEDs) can overcome the short comings of GPS. Furthermore LEDs offer many advantages over traditional incandescent and fluorescent light sources such as a longer life expectancy, high tolerance to humidity, high brightness, ecofriendliness and energy efficiency. [3].

Various LED-based indoor positioning systems exist in literature which are based on received signal strength (RSS) [4], time difference of arrival (TDOA) [5], angle of arrival (AOA) [6], image sensors [7], correlation [8], fingerprinting [9], analytically solving Lambertian transmission equations [10], heuristically dealing with light intensity distribution [11] and so on. RSS requires the knowledge of properties of optical channels among transmitter, receiver and the optical power transmitted by each LED which is difficult to achieve. TDOA based systems require a precise delay control to adjust the initial phase. The AOA approach, which is an unsuited choice for radio frequency (RF), is a promising technique in visible light communication (VLC), requires multiple receivers for location estimation. Image sensor based localization systems have issues of light flickering in addition to complex and relatively expensive hardware. Correlation and fingerprinting techniques require an offline training stage and if the indoor environment changes, a retraining is required.

In this paper, we present a beam scanning approach for indoor localization using LEDs and test it using experimentation. System modeling and system design (transmitter and receiver) are discussed in Section 2. Section 3 discusses the proposed algorithm followed by results in Section 4. Paper is concluded in Section 5.

\section{System Modeling and Design}

\subsection{System Modeling}

In order to establish a communication system, transmitter, channel and receiver need to be modelled. Light emitted from an LED follows a Lambert emission pattern [12] which is governed by Eq. (1).

$$
R(\phi)=\frac{n+1}{2 \pi} P_{T} \cos ^{n_{l}}(\phi)
$$

where $P_{T}$ is the power transmitted from the LED, $\phi$ is the angle between the source orientation vector and the vector pointing from source to the receiver, $n$ is the mode number of the radiation lobe which is $n=\frac{-\ln 2}{\ln \left(\cos \Phi_{1 / 2}\right)}$ where $\Phi_{1 / 2}$ is the LED view angle at half power. The transmitted optical power is given by Eq. (2).

$$
P_{T}=K_{m} \int_{\Delta_{\min }}^{\Delta_{\max }} \int_{0}^{2 \pi} \Phi_{e} d \theta d \lambda
$$

where $\Delta_{\min }$ and $\Delta_{\max }$ are calculated from photodiode sensitivity curve, typically in the range of visible light spectrum, $\Phi_{e}$ is the energy flux and $K_{m}$ is the maximum visibility. It is assumed that the receiver is facing vertically upwards. Furthermore, for a typical VLC channel, the distance $(d)$ between the transmitter and the receiver is quite large as compared to the photodiode detector area $\left(A_{R}\right)$, which makes the received signal irradiance constant over the photodiode detector area. With these assumptions, the VLC channel 
impulse response $h(t)$ can be approximated as a scaled and delayed Dirac delta function from $L E D_{i}$ for $\theta_{i} \leq F o V$ is given by Eq. (3) [13].

$$
h_{i}(t)=\left\{\frac{n+1}{2 \pi} \frac{\cos ^{n}\left(\phi_{i}\right) \cos \left(\theta_{i}\right) A_{R}}{d_{i}^{2}} \delta\left(t-\frac{d_{i}}{c}\right)\right\}
$$

where $\theta_{i}$ is the angle between the receiver orientation vector and vector pointing from receiver to the source, $F o V$ is the field of view of the receiver and $c$ is the speed of light. The received signal can be expressed by Eq. (4) [14].

$$
P_{R}(t)=P_{T} \sum_{i=1}^{k} s_{i}(t) \otimes h_{i}(t)+\mathrm{n}(\mathrm{t})
$$

where $s(t)$ is the modulated signal and $n(t)$ is the noise from the channel. In order to distinguish between the signals from each angle of the LED, time division multiplexing (TDM) [15] is used and frame structure for one period is shown in Fig. 1. At the end of each frame, the receiver will have the information about the received signal strength (RSS) of LED for each angle. In order to distinguish between the LED1 and LED2, different frequencies (i.e. $1 \mathrm{kHz}$ and $2.5 \mathrm{kHz}$ ) are used.

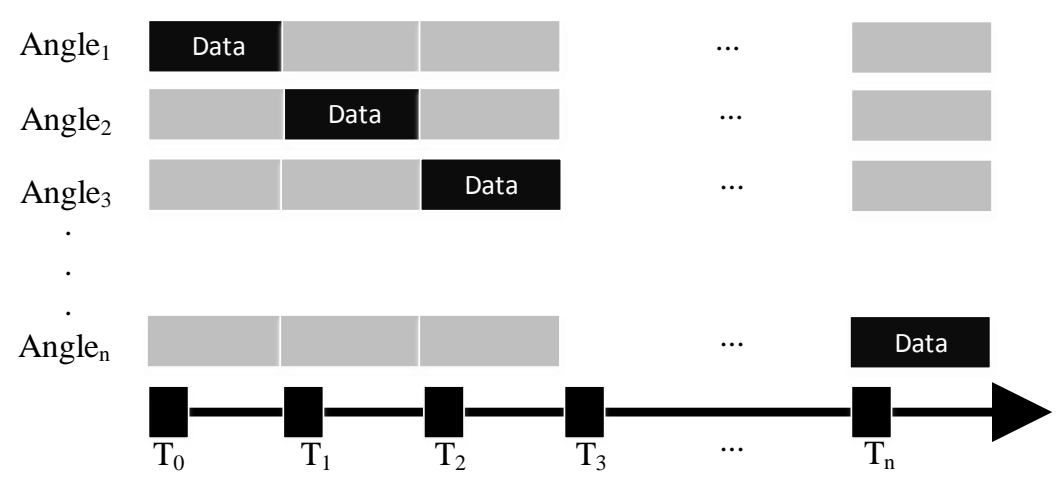

Fig. 1. Frame structure for one time slot.

\subsection{System Design}

\subsubsection{Transmitter design}

The block diagram of transmitter is shown in Fig. 2 and implementation details are shown in Fig. 3. It primarily consists of two units. The first part is the motor control unit and the second part is the data transmission unit. The motor control unit consists of stepper motor driver and stepper motor which is controlled by a microcontroller unit (MCU). The motor is connected to the LED lamp with the help of motor shaft. The data transmission part consists of signal generator which is responsible for modulating the signal. Angle information (as per Fig. 1) coming from MCU becomes one of the inputs of the multiplier and the other input is from the signal generator. The output of the multiplier is amplified with the help of an amplifier, which is fed to the LED driver circuit, followed by the LED lamp. 


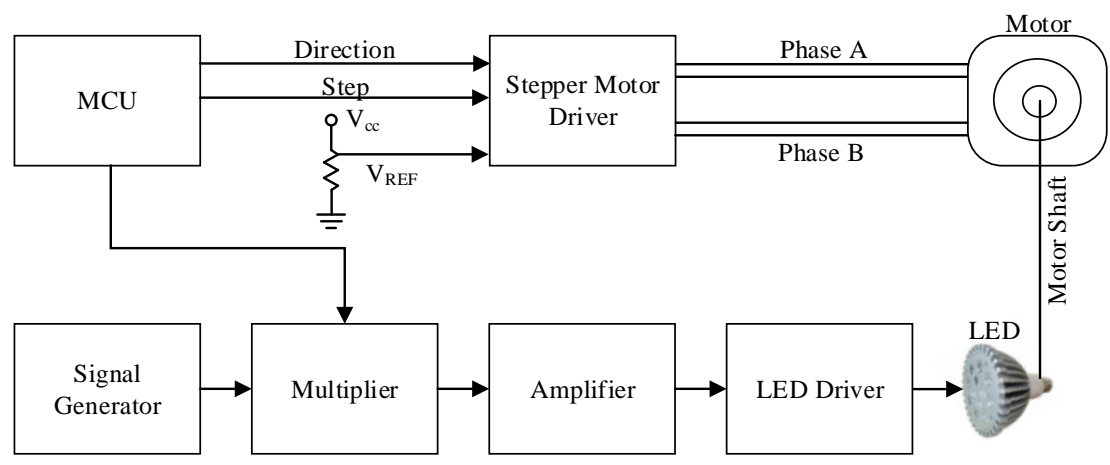

Fig. 2. Block diagram of transmitter.

Figure 3 shows the implementation details of the motor control unit and the experimental setup. Motor control unit consists of two major components; a voltage regulator and a stepping driver. In order to rotate the LED mounted on the stepper motor shaft to the desired location, a corresponding shift in angle is calculated. The stepping driver circuit calculates the number of steps required to rotate the LED to the desired position. For each step generated by the stepping driver circuit, the corresponding voltages are calculated by the voltage regulator circuit. For each step of the stepping motor, $1^{\circ}$ change of angel in LED is produced. A feedback signal generated from the stepper motor is fed back to the stepper motor to identify, if the desired shift in angle is achieved or not. If the desired angle is not achieved then the stepping driver again calculate the number steps required to achieve the desired shift in angle. This process keeps on repeating until the motor reaches to the desired angle.

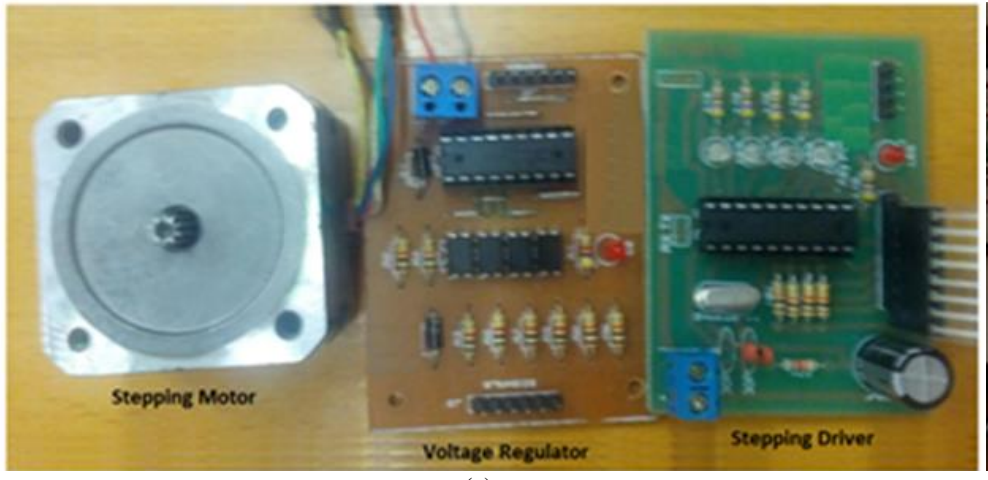

(a)

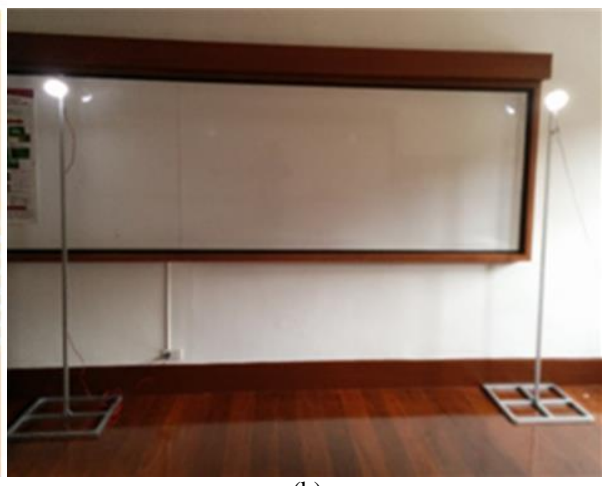

(b)

Fig. 3. (a). Motor control unit (b). Experimental setup.

\subsubsection{Receiver design}

Block diagram of the proposed receiver is shown in Fig. 4 and the implementation details are shown in Fig. 5. The first part of the receiver is a photodiode which is used to convert the modulated light signals into electric current. Photodiode is followed by a trans-impedance amplifier (TIA) which converts the electric signal into voltage. The output of the TIA becomes the input to the differential amplifier for adaptive minimum voltage cancellation. The purpose of the minimum voltage cancellation circuit is to remove the DC component and interference from the ambient light sources from the received signal. This is based on the calculation of minimum offset voltage of the received signal using a minimum voltage detection circuit [16]. Information from the minimum voltage canceller is received by the microcontroller and then sent to the computer for further processing. 


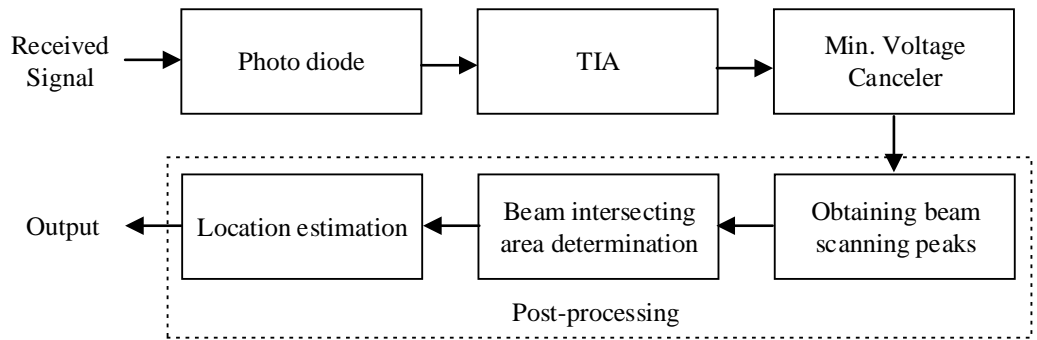

Fig. 4. Block diagram of the receiver circuit.

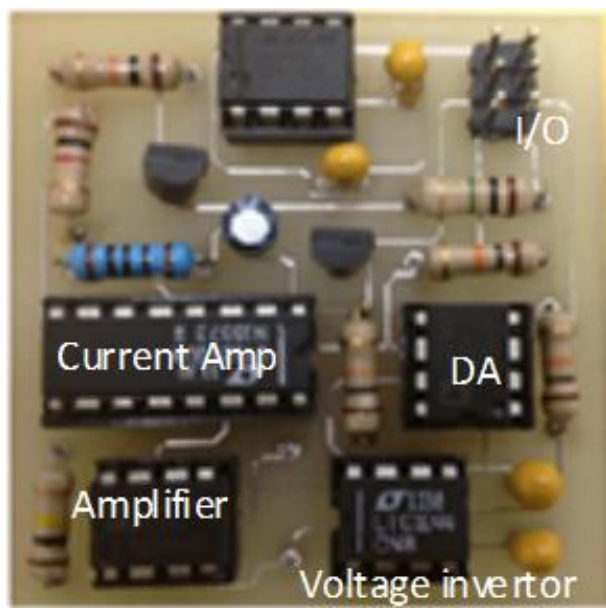

Fig. 5. Implementation of receiver circuit.

Details regarding the system parameters for a VLC link is tabulated in Table 1.

Table 1. System parameters for a VLC link.

\begin{tabular}{|l|l|l|}
\hline Entity & Parameter & Values \\
\hline Room & Dimensions & $3 \times 3 \times 3 \mathrm{~m}^{3}$ \\
\hline \multirow{4}{*}{ rransmitter } & No. of LEDs & 02 \\
\cline { 2 - 3 } & LEDs positions & $(0,0,2)(0,3,2)$ \\
\cline { 2 - 3 } & Step size & $8^{\circ}$ to $20^{\circ}$ \\
\cline { 2 - 3 } & Wattage & $12 \mathrm{~W}$ \\
\cline { 2 - 3 } & Viewing angle & $25^{\circ}$ \\
\hline \multirow{4}{*}{ Receiver } & No. of photodiode(s) & 01 \\
\cline { 2 - 3 } & Half sensitivity & $\pm 65^{\circ}$ \\
\cline { 2 - 3 } & Radiant surface area & $7.5 \mathrm{~mm}^{2}$ \\
\hline
\end{tabular}

\section{The Proposed Algorithm}

For this work, we consider a case in which two-dimensional (2-D) localization needs to be determined. Two transmitters (LED1, LED2) are used. Each LED $L_{i}$ is mounted above the localization area with the centre of the LED emission focused at $\left(\frac{x_{-} a x i s_{M A X}}{2}, 0\right)$ for LED1 and $\left(\frac{x_{-} a x i s_{M A X}}{2}, y_{-} a x i_{M A X}\right)$ for LED2 which are used as reference angles. The LEDs are pointed downwards facing the area with the elevation angle of $\theta_{e, i}$. The beam emitted with $\theta_{e, i}$ will have its projection in a conical geometry on 2D plane and its vertex is defined as a location of a virtual point source. The scenario is shown in Fig. 6. 


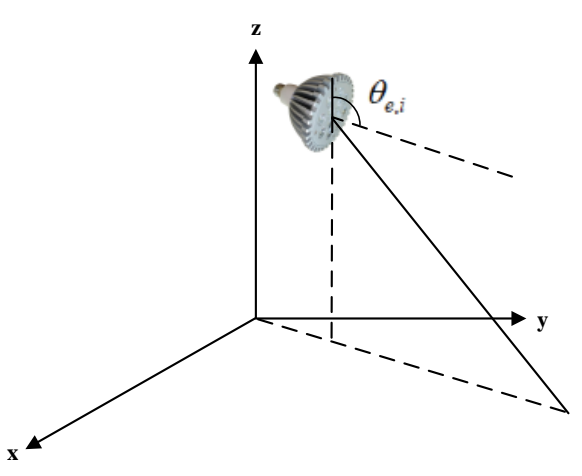

(a)

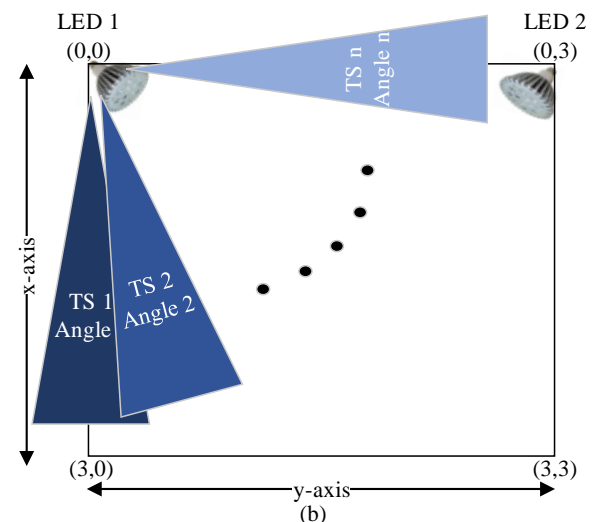

(b)

Fig. 6. (a). Parameters of LED (b). Beam scanning process.

The proposed algorithm is explained in the following three steps.

\section{(A). Beam scanning and higher peaks recording}

In the first step, the LED1 is moved with a step of few degrees $\left(8^{\circ}\right.$ to $\left.20^{\circ}\right)$ from a reference point to an end point. $0^{\circ}$ refers to the data transmission at first time slot (TS) and $90^{\circ}$ refers to last TS data transmission which is shown in Fig. 6(b). During the scanning process, LED records the highest and the second highest intensity with their respective time slot which tells the information about the angle of the LED. The same procedure is applied to LED2 as well.

\section{(B). Finding an intersecting area}

When the receiver has the knowledge at which angle of the LED, the receiver receives the maximum values, the intersecting area between the two LED beams can be found. The two RSS values from each LED will always be consecutive and the four points (two highest readings from LED1 and two from LED2) will correspond to a specific region in a $2 \mathrm{D}$ plane where the object of interest is. This area refers to the intersecting area of the beams from LED1 and LED2 which is depicted in Fig. 7 and the area can simply be constructed by joining the point of points where LEDs have the maximum values.

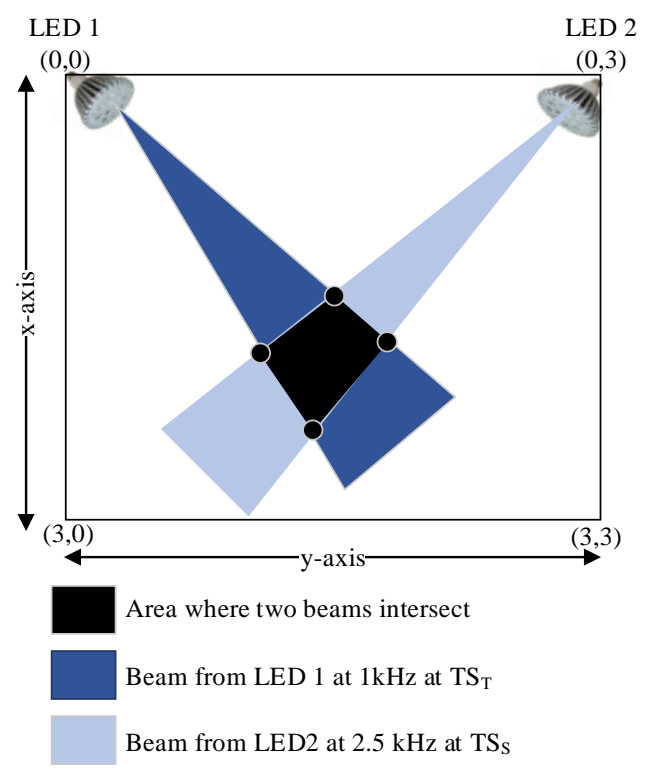

Fig. 7. Determining intersecting area of two beams. 


\section{(C). Finding the location of an object}

When the area under the intersecting beams is known, the next step is to find the location of an object. For position estimation, the bicubic spline interpolation is used [17]. Interpolation is a technique for creating new data points within the range of a discrete set of known data points and a bicubic spline is a special case of bicubic interpolation. The intensity at the corners of the intersecting area are known to the receiver (during the offline stage, these readings are measured and recorded in the lookup table of the receiver). The advantage of using bicubic spline interpolation is that instead of using four intensity values to estimate the position, bicubic spline interpolation gives 16 points which can be processed to approximate the position. To understand this, one can consider Fig. 8.

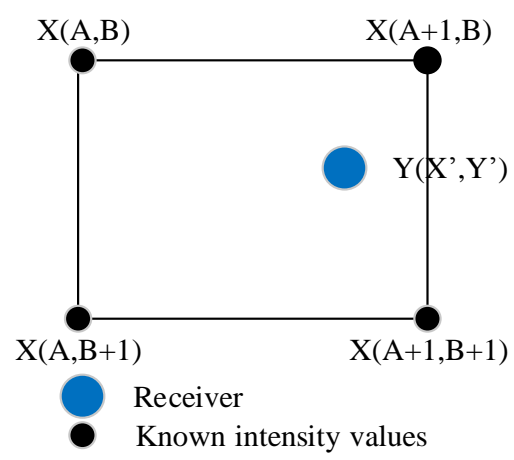

Fig. 8. Bicubic spline interpolation for our case

The original intensity values are $f(A, B), f(A+1, B), f(A, B+1), f(A+1, B+1)$. By taking the partial derivatives along the $x$-axis, $y$-axis and cross derivatives of each of these will give 16 points in total. It is worth mentioning here that a VLC channel is comparatively static and light intensity is changed in a known fashion with respect to the distance. Furthermore, ignoring the effect of multipath, reflection and shadowing (which is insignificant for a typical official environment), the equations for bicubic spline interpolation can be modified to the following set of equations.

$$
\begin{aligned}
& f(x, y)=\sum_{i=1}^{4} \sum_{j=1}^{4} x^{i} y^{j} \\
& \partial_{x} f(x, y)=\sum_{i=1}^{4} \sum_{j=1}^{4} i x^{i-1} y^{j} \\
& \partial_{y} f(x, y)=\sum_{i=1}^{4} \sum_{j=1}^{4} j x^{i} y^{j-1} \\
& \partial_{x y} f(x, y)=\sum_{i=1}^{4} \sum_{j=1}^{4} i j x^{i-1} y^{j-1}
\end{aligned}
$$

With the help of these 16 points, the position of an object is estimated.

\section{Results}

For experimental demonstration, we took two off-the-shelf, narrow beam, 12 W LED lamps with viewing angle of $25^{\circ}$. A high speed photo diode is used with angle of half sensitivity equals to $\pm 65^{\circ}$ and the radiant surface area of $7.5 \mathrm{~mm}^{2}$. Each LED lamp is composed of 12 LEDs which are arranged in a circular fashion. The height of the LED lamps were fixed at $2 \mathrm{~m}$ and room dimensions are kept to be $3 \times 3 \times 2 \mathrm{~m}^{3}$. The horizontal distance between the LEDs is $3 \mathrm{~m}$ and the position of the LED lamps are fixed and known. For the given scenario, we evaluate the performance of the proposed localization algorithm for noisy and noisefree environment. We also investigate the effect of LED rotation step size on location estimation. 


\subsection{Performance Evaluation under Noise-Free and Noisy Environment}

For the first case, light from all the ambient light sources such as sun light, fluorescent and incandescent light sources etc. are not allowed to enter in the experimental area and only the lights from the LEDs contributing to this experiment are turned on. LEDs are moved at various step sizes and the mean location estimation error is plotted against each step size. It can be observed from Fig. 9 that as the step size increases, the accuracy of location estimation decreases implying inverse relation between step size and localization errors. For this experiment, the minimum step size for LED movement is kept at $8^{\circ}$ however it can be further reduced. At $8^{\circ}$, the position estimation error for noise-free case is $13 \mathrm{~cm}$ which is superior to conventional RF and VLC localization techniques.

The second graph (green line) in Fig. 9 is for the case when receiver is placed in an environment where sun light can enter in the room (not direct sun light) and ambient light sources are also present which results in generation of shot noise. For moderate conditions, the value of shot noise is typically around 160 $\mathrm{dbm}$ considering low data transmission which is true for our case [18]. Under this condition, for $8^{\circ}$ step size, the localization error is $18 \mathrm{~cm}$ and for $20^{\circ}$ the localization error is approximately $45 \mathrm{~cm}$.

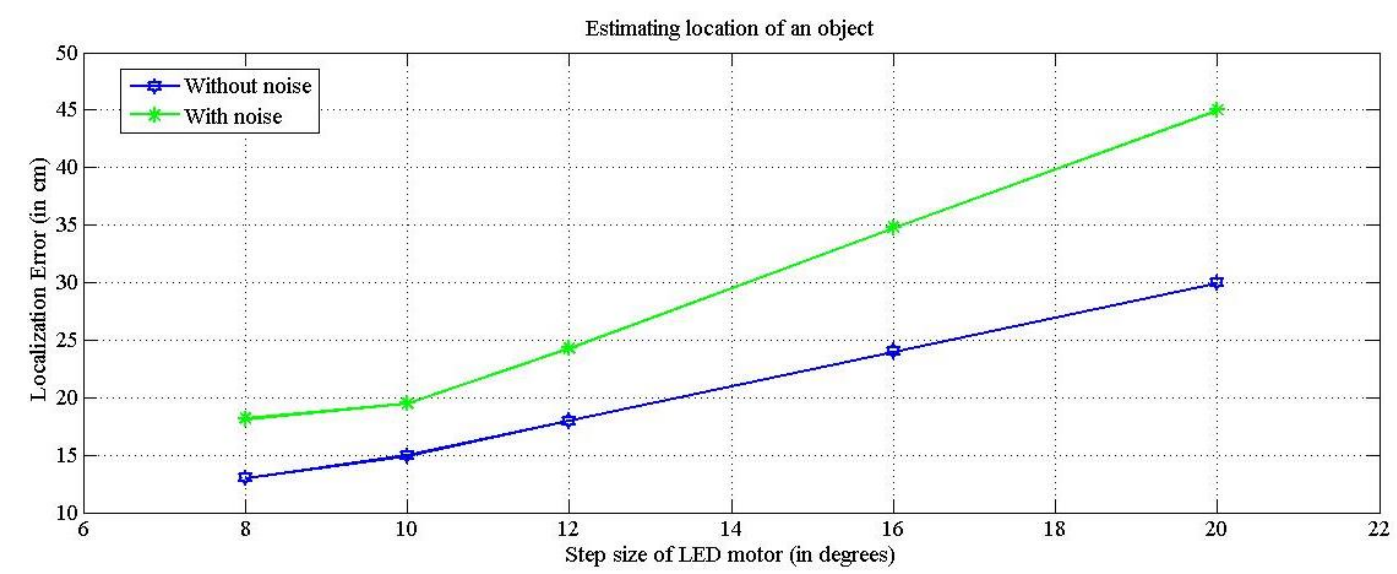

Fig. 9. Localization error at different step sizes for a no noise and noisy environment

\subsection{Location-wise Positioning Estimation Error}

Further analysis is done for the location estimation error with respect to the coordinates of the room. It has been observed that the location estimation error at the corners is more as compared to the center of the room which is because of the directionality of Lambert emission transmission. When the receiver is near to the corner, the received intensity from the diagonal source can be considerably degraded and is susceptible to noise interferences. However for the proposed algorithm, the location estimation error primarily depends on the overlapping area of the beams of the two LED lamps. If the intersecting area (as shown in Fig. 7) is bigger, then the error will also be more and vice versa. The corners which are near the LEDs will have small intersecting area and as the beam goes farther, the intersecting area increases which results in uneven location estimation errors at the corners. Figure 10 shows the positioning error for a specific case when the step angle of the LEDs is $8^{\circ}$ and the system is noise-free (no sunlight or ambient light sources). 


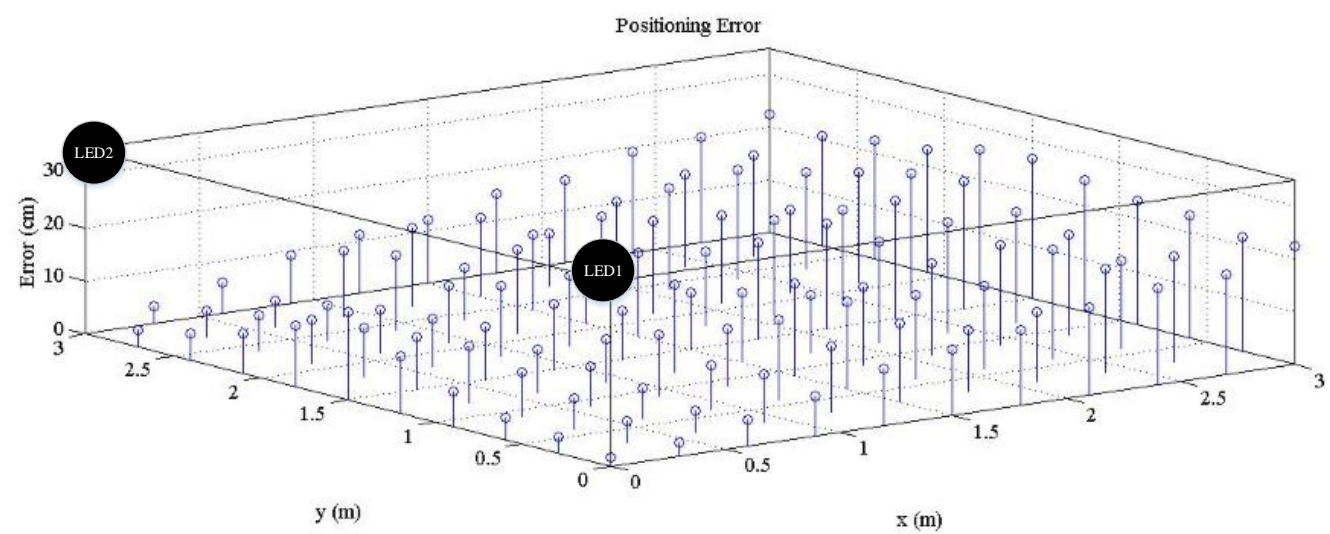

Fig. 10. Location-wise positioning estimation error

In this work, the database used for estimating the position of an object using beam scanning is primarily based on the received signal strength from the two LEDs. However it is worth investing what will be the effect of the position accuracy if angle of arrival is used instead of received signal strength for database creation. Angle of arrival is a promising technique for indoor localization using light emitting diodes, however its performance with respect to beam scanning is yet to be explored.

\section{Conclusion}

In this paper, we propose an LED beam scanning method for indoor localization. In the developed system, probable location of an object is attained by intersecting two conic sections projected by two LEDs beams. LEDs are modulated at different frequencies and moved with the help of a motor at fixed and known angles so that each LED can cover the whole area of interest. Receiver estimates the beam direction of the LED by determining at which time slot the data is received. After receiving the two highest values of signal strength from each LED, the intersecting area of two beams is determined. A bicubic spline interpolation is applied for approximating the location of an object. Results show that the proposed algorithm offers a precision up to $13 \mathrm{~cm}$ even in the presence of moderate noise. Therefore, the proposed algorithm is apposite candidate for localization technique for indoor visible light communication system.

\section{References}

[1] J. Armstrong, Y. Sekercioglu, and A. Neild, "Visible light positioning: A roadmap for international standardization," Communications Magazine, IEEE, vol. 51, no. 12, pp. 68-73, 2013.

[2] M. Saadi, L. Wattisuttikulkij, Y. Zhao, and P. Sangwongngam, "Visible light communication: Opportunities, challenges and channel models," International Journal of Electronics \& Informatics, vol. 2, no. 1, pp. 1-11, 2013.

[3] M. Saadi, A. Bajpai, Y. Zhao, P. Sangwongngam, and L. Wuttisittikulkij, "Design and implementation of secure and reliable communication using optical wireless communication," Frequen₹, vol. 68, no. 1112, pp. 501-509, 2014.

[4] S. Y. Jung, S. R. Lee, and C. S. Park, "Indoor location awareness based on received signal strength ratio and time division multiplexing using light-emitting diode light," Optical Engineering, vol. 53, no. 1 016106-1-016106-5, 2014.

[5] S. Y. Jung, S. Hann, and C. S. Park, "TDOA-based optical wireless indoor localization using LED ceiling lamps," IEEE Transactions on Consumer Electronics, vol. 57, no. 4, pp. 1592-1597, 2011.

[6] S. H. Yang, H. S. Kim, Y. H. Son, and S. K. Han, "Three-dimensional visible light indoor localization using AOA and RSS with multiple optical receivers," Journal of Lightwave Technology, vol. 32, no. 14, pp. 2480-2485, 2014.

[7] M. S. Hossen, Y. Park, and K. D. Kim, "Performance improvement of indoor positioning using lightemitting diodes and an image sensor for light-emitting diode communication," Optical Engineering, vol. 54, no. 4, 2015. 
[8] S. Y. Jung, S. Hann, S. Park, and C. S. Park. "Optical wireless indoor positioning system using light emitting diode ceiling lights," Microwave and Optical Technology Letters, vol. 54, no. 7, pp. 1622-1626, 2012.

[9] J. Vongkulbhisal, B. Chantaramolee, Y. Zhao, and W. S. Mohammed, "A fingerprinting-based indoor localization system using intensity modulation of light emitting diodes," Microwave and Optical Technology Letters, vol. 54, no. 5, pp. 1218-1227, 2012.

[10] Z. Zhou, M. Kavehrad, and Peng Deng, "Indoor positioning algorithm using light-emitting diode visible light communications," Optical Engineering, vol. 51, no. 8, pp. 085009-1-085009-6, 2012.

[11] M. Saadi, Y. Zhao, L. Wuttisittikulkij, and T. A. Khan, "A heuristic approach to indoor localization using light emitting diodes," Journal of Theoretical and Applied Information Technology, vol. 84, no. 3, pp. 332-338, 2016.

[12] J. Grubor, S. Randel, K. D. Langer, and J. W. Walewski, "Broadband information broadcasting using LED-based interior lighting," Journal of Lightwave Technology, vol. 26, no. 24, pp. 3883-3892, 2008.

[13] Z. Ghassemlooy, W. Popoola, and S. Rajbhandari, Optical Wireless Communications System and Channel Modelling with Matlabß. CRC Press, 2012.

[14] M. B. Rahaim, T. Borogovac, and J. B. Carruthers, "CandlES: Communication and lighting emulation software," in Proceedings of the fifth ACM international workshop on Wireless network testbeds, experimental evaluation and characterization, 2010, pp. 9-14.

[15] N. Elliot, "Time division multiplexing," U.S. Patent 3,599,160, 1971.

[16] Y. Zhao and J. Vongkulbhisal, "Design of visible light communication receiver for on-off keying modulation by adaptive minimum-voltage cancelation," Engineering Journal, vol. 17, no. 4, pp. 125-130, 2013.

[17] C. De Boor, C. De Boor, E. U. Mathématicien, C. De Boor, and C. De Boor, A Practical Guide to Splines, vol. 27. New York: Springer-Verlag, 1978, p. 325.

[18] M. Wolf and K. Dieter, "Short-range wireless infrared transmission: The link budget compared to RF,” IEEE Wireless Communications, vol. 10, no. 2 (2003): 8-14. 